\title{
Indolicidin Targets Duplex DNA: Structural and Mechanistic Insight through a Combination of Spectroscopy and Microscopy
}

\author{
Anirban Ghosh, ${ }^{[a]}$ Rajiv Kumar Kar, ${ }^{[a]}$ Jagannath Jana, ${ }^{[a]}$ Abhijit Saha, ${ }^{[b]}$ Batakrishna Jana, ${ }^{[b]}$ \\ Janarthanan Krishnamoorthy, ${ }^{[c]}$ Dinesh Kumar, ${ }^{[d]}$ Surajit Ghosh, ${ }^{*[b]}$ Subhrangsu Chatterjee, ${ }^{*[a]}$ \\ and Anirban Bhunia*[a]
}

\begin{abstract}
Indolicidin (IR13), a 13-residue antimicrobial peptide from the cathelicidin family, is known to exhibit a broad spectrum of antimicrobial activity against various microorganisms. This peptide inhibits bacterial DNA synthesis resulting in cell filamentation. However, the precise mechanism remains unclear and requires further investigation. The central PWWP motif of IR13 provides a unique structural element that can wrap around, and thus stabilize, duplex B-type DNA structures. Replacements of the central Trp-Trp pair with Ala-Ala, His-His, or Phe-Phe residues in the PxxP motif significantly affects the ability of the peptide to stabilize duplex DNA. Results of microscopy studies in conjunction with spectroscopic data confirm that the DNA duplex is stabilized by IR13, thereby inhibiting DNA replication and transcription. In this study we provide high-resolution structural information on the interaction between indolicidin and DNA, which will be beneficial for the design of novel therapeutic antibiotics based on peptide scaffolds.
\end{abstract}

Bacterial resistance toward antibiotics poses a significant challenge in the treatment of many deadly infectious diseases. ${ }^{[1]}$ To counteract the emergence of multidrug-resistant (MDR) bacterial strains, an intensive search for a new generation of antibiotics that closely resemble the host defense cationic antimicrobial peptides (CAMPs, a key component in innate immunity), has been initiated. ${ }^{[2-6]}$ Various types of natural CAMPs or synthetic analogues of antimicrobial peptides (AMPs) ${ }^{[7-10]}$ have shown broad-spectrum antibiotic activity, meeting the current demand for a novel class of effective antibiotics. ${ }^{[11-15]}$ In this

\footnotetext{
[a] A. Ghosh, R. K. Kar, J. Jana, Dr. S. Chatterjee, Dr. A. Bhunia

Department of Biophysics, Bose Institute

P-1/12 CIT Scheme VII (M), Kolkata 700054 (India)

E-mail: subhrangsu@gmail.com bhunia@jcbose.ac.in

[b] A. Saha, B. Jana, S. Ghosh

Chemistry Division, CSIR-Indian Institute of Chemical Biology (IICB)

4, Raja S. C. Mullick Road, Kolkata-700032 (India)

E-mail:sghosh@iicb.res.in

[c] J. Krishnamoorthy

Department of Chemistry and Biophysics

University of Michigan, Ann Arbor, MI 48109-1055 (USA)

[d] D. Kumar

Centre of Biomedical and Magnetic Resonance (CBMR)

SGPGIMS Campus, Raibareli Road, Lucknow (India)

Supporting information for this article is available on the WWW under http://dx.doi.org/10.1002/cmdc.201402215.
}

context, indolicidin, a 13-residue AMP rich in tryptophan (ILPWKWPWWPWRR-NH $\mathrm{N}_{2}$; hereafter referred to as IR13; Figure $1 \mathrm{a}$ ) is a promising precursor molecule for drug design due

$$
\begin{array}{ll}
\text { ILPWKWPWWP } & \text { WRR }-\mathrm{NH}_{2} \\
\text { ILPWKWPAAP } & \text { WRR }-\mathrm{NH}_{2} \\
\text { ILPWKWP FFP } & \text { WRR }-\mathrm{NH}_{2} \\
\text { ILPWKWP HHP } & \text { WRR }-\mathrm{NH}_{2}
\end{array}
$$

b)

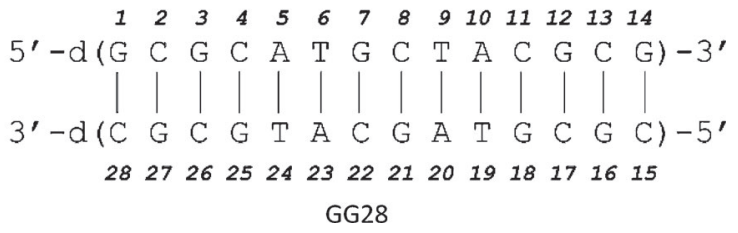

Figure 1. a) Sequence of indolicidin (IR13) and its analogues (IR13AA, IR13FF and IR13HH) in which Trp8 and Trp9 are replaced by Ala-Ala, Phe-Phe, and His-His residues, respectively. b) Sequence of GC-rich duplex DNA (GG28).

to its activity against a wide variety of Gram-positive and Gram-negative bacteria, fungi, and protozoa. ${ }^{[16]}$ Structurally, IR13 is one of the smallest AMPs. It is natively disordered in aqueous solution, but in lipid micelle environments such as those of DPC and SDS, it adopts a wedge-shaped conformation. ${ }^{[17]}$ A recent study by Shaw et al. demonstrated that IR13 must interact with the cell membrane prior to membrane permeabilization or subsequent inhibition of DNA synthesis. ${ }^{[18]}$ It is well known that the mode of action of IR13 is through inhibition of bacterial DNA replication and transcription. ${ }^{[19]}$ However, it remains to be determined just how IR13 binds DNA and disrupts the transcription process. To address this key question, we combined the results from several low-resolution techniques such as micropatterned surface chemistry microscopy, spectroscopic techniques such as fluorescence and CD, in conjunction with high-resolution NMR spectroscopy. In so doing, we were able to determine the structural details behind the interaction between IR13 at DNA at atomic resolution.

For this study we chose an oligonucleotide sequence in which both $G: C$ and A:T base pairs are present (GG28; Figure $1 \mathrm{~b}$ ), so as to compare the preferential interaction of such base pairs with IR13. Complementarily, the propensity of IR13 to recognize different base pairs in duplex DNA was also stud- 
ied by varying the sequence motif PWWP in IR13 (Figure $1 \mathrm{a}$ ), as the PWWP motif is known to recognize DNA and form a helical turn structure. ${ }^{[20,21]}$

As DNA nucleobases and Trp residues in the peptide fluoresce in the same spectral region, FITC labeling of the peptide helps to identify and differentiate the fluorescence emission that comes exclusively from the peptide for studying the DNA-peptide interaction. Titration of GG28 into a solution containing FITC-labeled IR13 peptide showed an enhancement in the fluorescence emission intensity for FITC. This enhancement is due to aggregation of IR13 in the aqueous medium. The characteristic behavior of IR13 in the aggregated form was previously demonstrated by dynamic light scattering (DLS) in the aqueous medium. ${ }^{[22]}$ The 1D ${ }^{1} \mathrm{H}$ NMR spectrum of IR13 also confirms aggregation of the peptide in aqueous solution through line broadening of up to $15-18 \mathrm{~Hz}$ (Supporting Information, Figure S1). The occurrence of seven peaks for five indole $\mathrm{NH}$ protons is due to cis-trans isomerism of the XP unit (where $\mathrm{x}=$ any amino acid).

Titration of GG28 into solution containing peptide yielded a biphasic curve with a classical hyperbolic profile at lower concentrations and a sigmoidal profile at the higher concentration. The estimated dissociation constant $\left(K_{\mathrm{D}}\right)$ for the initial binding profile was $3.3 \pm 0.2 \mu \mathrm{M}$, which signifies an initial noncooperative form of binding between the DNA and the peptide (Figure $2 \mathrm{a}$ ). However, further addition of GG28 led to cooperative binding of DNA to FITC-labeled IR13 (showing a sigmoidal fit), with an estimated $K_{\mathrm{D}}$ value of $\sim 11.2 \pm 0.1 \mu \mathrm{M}$ (Figure $2 \mathrm{a}$ ). A previous surface plasmon resonance (SPR) study of the DNA-IR13 interaction showed a $K_{\mathrm{D}}$ value for complex formation at $\sim 40 \mu \mathrm{M},{ }^{[23]}$ which is closer to the product of both $K_{\mathrm{D}}$ values estimated in this study (i.e., $3.3 \times 11.2=36.9 \mu \mathrm{M}$ ), indicating a combination of both non-cooperative and cooperative binding of DNA to IR13. The CD spectra for GG28 and the GG28-IR13 complex consist of positive maxima at $\sim 275 \mathrm{~nm}$ and negative minima at $\sim 255 \mathrm{~nm}$ (Figure $2 \mathrm{~b}$ ). The secondary structure of DNA does not change significantly even after binding to the peptide, thereby retaining its native B-form. To study further how IR13 interacts with GG28 we decided to use our recently developed micropatterned surface technique using fluorescence microscopy. ${ }^{[24,25]}$ For this, we attached two fluorophores, biotin and rhodamine, at the $5^{\prime}$-end of each of the two single-stranded GG28 oligonucleotides (Supporting Information, Scheme S1A). The tagged duplex GG28 was immobilized onto the neutravidin-loaded biotin-micropatterned surface through the biotin-neutravidin-biotin interaction (Figure $2 \mathrm{c}$ and Figure S2) for $10 \mathrm{~min}$. Unbound excess FITC-IR13 was removed by repeated washing with buffer. Microscopy images at $\lambda 561$ and $488 \mathrm{~nm}$ reveal red and green micropatterned surfaces for GG28 and IR13, respectively (Figure 2 c,d); the co-localization of both is identified by the yellow merged pattern (Figure 2e), indicating the interaction of IR13 with immobilized GG28 present on the square blocked micropatterned surface. To determine whether IR13 binds the micropatterned surface directly or through DNA, we performed a control experiment in which avidin-rhodamine dye was incubated with the micropatterned surface and subsequently loaded with a)

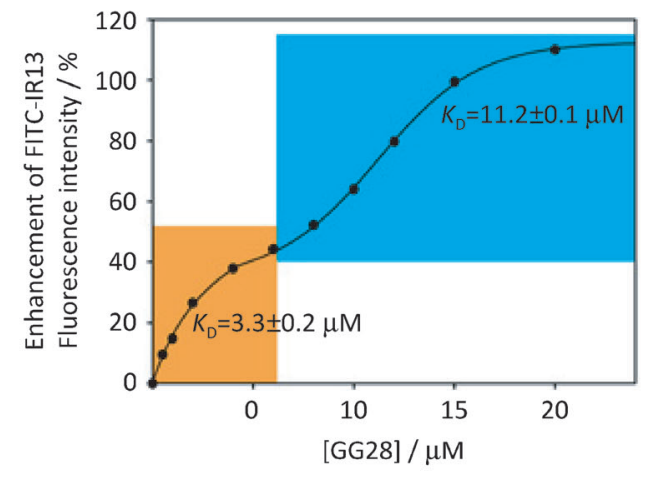

b)

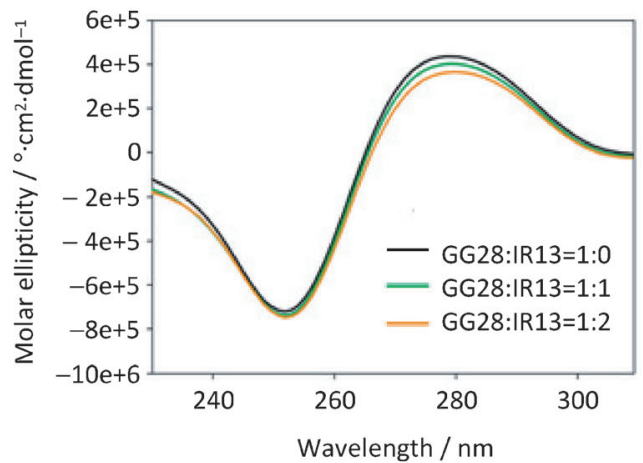

c) d)

e)

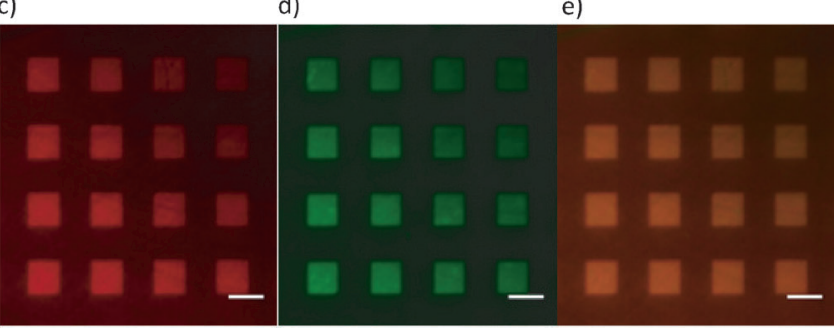

Figure 2. Interaction of IR13 and DNA in solution and on a biotin-micropatterned surface. a) Increase in the fluorescence intensity profile of FITC-labeled IR13 $(10 \mu \mathrm{M})$ as a function of increasing titrated concentrations of duplex DNA, GG28 (up to $25 \mu \mathrm{M}$ ). b) Secondary structure elucidated by CD spectroscopy for the interaction of GG28 and IR13. CD spectra of GG28 $(10 \mu \mathrm{M})$ titrated with IR13 at concentrations ranging from 5 to $20 \mu \mathrm{M}$. c) Red micropatterned surface indicates the immobilization of Biotin-rhodamineDNA on a biotin-micropatterned surface through a biotin-neutravidin-biotin interaction. d) Green micropatterned surface indicates the binding of FITC-labeled IR13 peptide on a micropatterned surface. e) Yellow merged image confirms that the FITC-IR13 peptide specifically binds to biotin-rhodamine labeled DNA immobilized on a biotin-micropatterned surface. Scale bars: $10 \mu \mathrm{m}$.

FITC-IR13 for observation by inverted microscopy. The resulting images show the binding of the avidin-rhodamine dye to the micropattern, but not the FITC-IR13 peptide (Supporting Information, Figure S3). The above results clearly indicate that the FITC-IR13 peptide specifically binds to the micropattern only through DNA.

We also tested the cellular uptake of IR13 by A549 lung cancer cells. Surprisingly, we observed that FITC-IR13 can localize not only in the cytoplasm (Figure $3 \mathrm{a}$ ) but in the nuclei of A549 cells as well (Figure $3 \mathrm{~b}$ ). The combined images of FITCtagged IR13 (green) and DAPI-stained nuclei (blue) clearly indi- 
a)

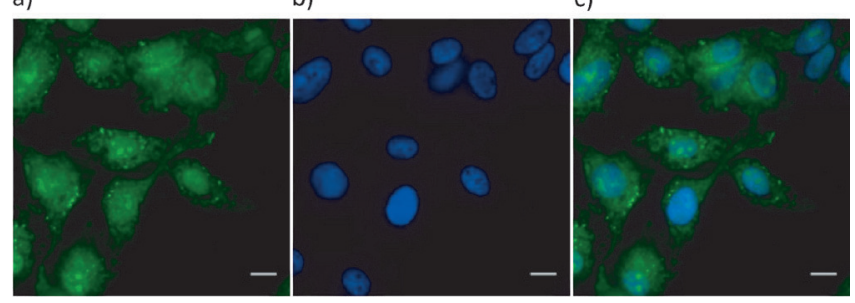

Figure 3. Uptake of FITC-IR13 peptide into A549 lung cancer cells. a) Green indicates the uptake of FITC-IR13 peptide into cells. b) Blue indicates nuclear staining by DAPI. c) Merged image shows that the FITC-IR13 peptide penetrates into cell nuclei. Scale bars: $10 \mu \mathrm{m}$.

cate that IR13 diffuses into the nuclei of cancer cells and directly interacts with the DNA (Figure $3 \mathrm{c}$ ).

Additional evidence for binding of IR13 to GG28 was obtained from thermal denaturation studies carried out with $C D$ and NMR spectroscopy. It is well known that the double-helical structure of DNA is quite stable due to hydrogen bonding and base stacking interactions. Thus, the thermal denaturation behavior of GG28 in the presence of IR13 or its analogues (Figure 1 a) can provide useful information about the configuration and kinship of the DNA-peptide interactions. The melting temperature $\left(T_{\mathrm{m}}\right)$ of the control DNA GG28 was found to be $52.2 \pm$ $0.7^{\circ} \mathrm{C}$ (Figure $4 \mathrm{a}$ ), whereas, the $T_{\mathrm{m}}$ of the GG28-IR13 complex was increased to $63.2 \pm 1.0^{\circ} \mathrm{C}$ (Figure $4 \mathrm{a}$ ). This clearly shows that the DNA-peptide complex is thermodynamically more stable than free DNA. In another case, GC28 [d(GCGCATATTAATGC) $)_{2}$ (Supporting Information, Scheme S1B) was also investigated to determine whether the degree to which IR13 stabilizes DNA duplexes is a function of their sequence. The $T_{\mathrm{m}}$ determined by CD for GC28 in complex with IR13 was found to be $50.6{ }^{\circ} \mathrm{C}$, whereas $T_{\mathrm{m}}$ for native $\mathrm{GC} 28$ was $40.1{ }^{\circ} \mathrm{C}$ (Figure S4). These data reflect that the sequence of $\mathrm{GC} 28$ does not significantly affect its binding to IR13. Notably, the PWWP motif is responsible for DNA recognition, ${ }^{[20,21]}$ and so to investigate the sequence dependence of the PWWP motif on DNA binding, we replaced the extended aromatic system of the Trp-Trp dipeptide with Phe-Phe, His-His, or Ala-Ala, consequently decreasing the surface area and hydrophobicity. We premised that increasing the hydrophobicity and surface area

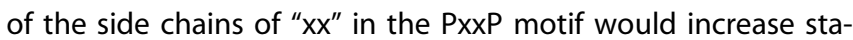
bilization of the DNA structure (Figure $4 \mathrm{a}$ and inset). Results from the profile of micropatterned surface chemistry validated this assumption in that the FITC-IR13 analogue containing the nonaromatic dipeptide Ala-Ala instead of Trp-Trp (FITCIR13AA), did not show any green micropatterned surface (Figure $4 \mathrm{~b}-\mathrm{d}$ ). This clearly demonstrates that the bulkier and hydrophobic Trp-Trp pair in IR13 plays a significant role in binding GG28.

These results motivated us to look further into the atomiclevel interaction of IR13 with GG28 by using NMR spectroscopy. To evaluate the contribution to base-pairing stability by imino protons in duplex DNA, a melting study with control DNA and a DNA-peptide complex was performed. The chemical-shift-based melting profile showed that the thymine N3

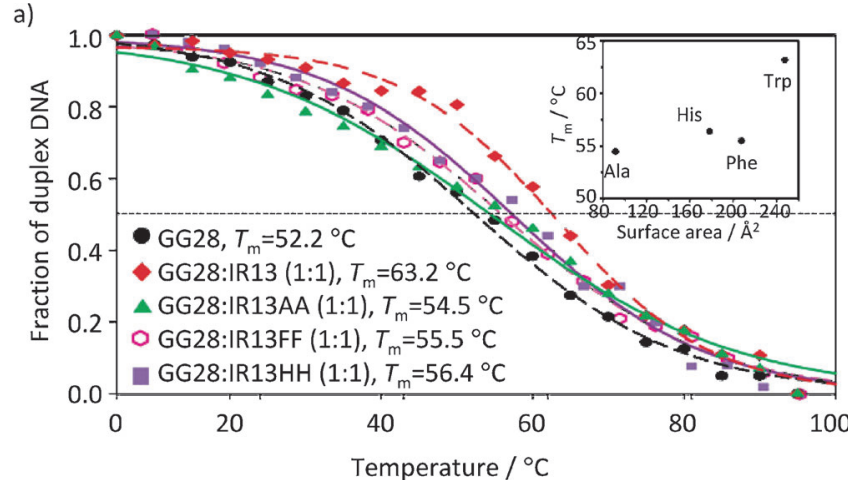

b) c)

d)

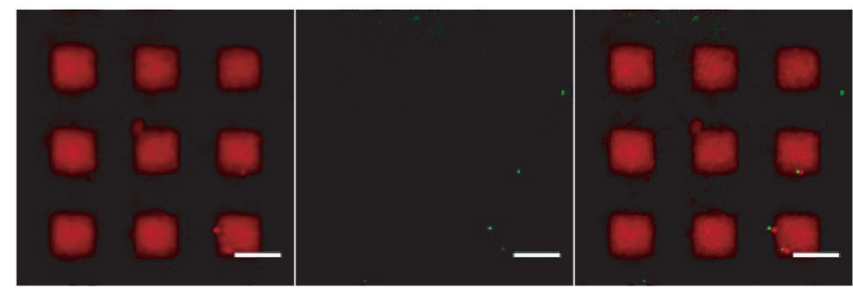

Figure 4. Interaction of IR13 and IR13AA with DNA. a) CD melting curve of GG28 in the absence (black) and in the presence of IR13 (red), IR13AA (green), IR13FF (violet), and IR13HH (purple). The inset shows that the $T_{\mathrm{m}}$ of GG28 is increased in the complexed form with increasing surface area of the xx motif in the PxxP sequence of IR13 analogues. b) Red micropatterned surface indicates that biotin-rhodamine-DNA is immobilized on a biotin-micropatterned surface through the biotin-neutravidin-biotin interaction.

c) The absence of a green micropatterned surface indicates that FITC-labeled IR13AA peptide did not bind the surface. d) Merged image of panels b) and c) also indicates that the FITC-IR13AA peptide did not bind to immobilized biotin-rhodamine-labeled DNA on the micropatterned surface. Scale bars: $10 \mu \mathrm{m}$.

imino protons are more disordered than the guanine $\mathrm{N} 1$ imino protons of free GG28. Interestingly, there is a significant decrease in the melting profiles of thymine and guanine imino protons of GG28-IR13. The thymine imino protons are more affected than those of guanine in the melting profile (Figure $5 \mathrm{a}$ ). A slow increase in temperature from 15 to $55^{\circ} \mathrm{C}$ for the free DNA showed that the imino protons of thymines T6, T9, T19, and T24 were readily denatured relative to those of all the guanines, except G16 and G27, which were present in the terminal part of the DNA duplex (Figure $5 \mathrm{a}$ ). However, the same thymine imino protons in the complex form showed a greater stabilization over the course of the melting experiment, indicating a general stabilization of the core region of DNA in the presence of IR13. Significant perturbation in the chemical shifts of the cross-peaks such as T9-A20, T19-A10, T24-A5, and T6A23 were detected in the $2 \mathrm{D}{ }^{1} \mathrm{H}-{ }^{1} \mathrm{H}$ NOESY spectrum of GG28 upon addition of IR13 (Figure 5 b). In contrast, no such chemical shift perturbation was observed for the imino protons of guanines in GG28 complexed with IR13, suggesting IR13 preferentially interacts with the A:T region of the DNA (Figure $5 \mathrm{~b}$ ). This result clearly suggests that base-pair breathing in A:T regions is more pronounced than in $\mathrm{G}: \mathrm{C}$ regions.

We reached the same conclusion from an independent Amber-ff99SB force-field-based molecular dynamics (MD) simu- 
GG28

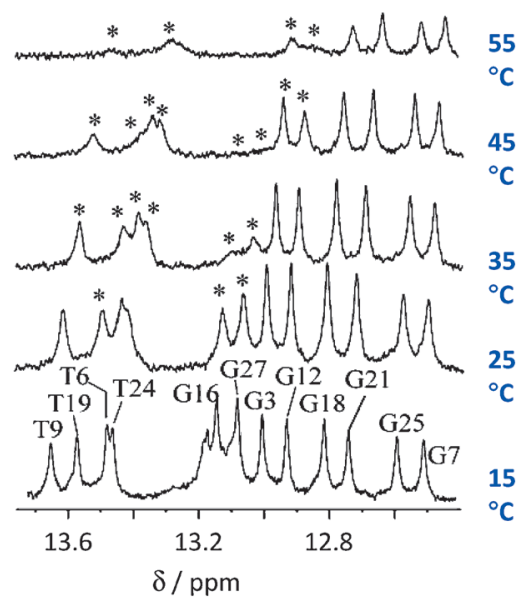

GG28+IR13
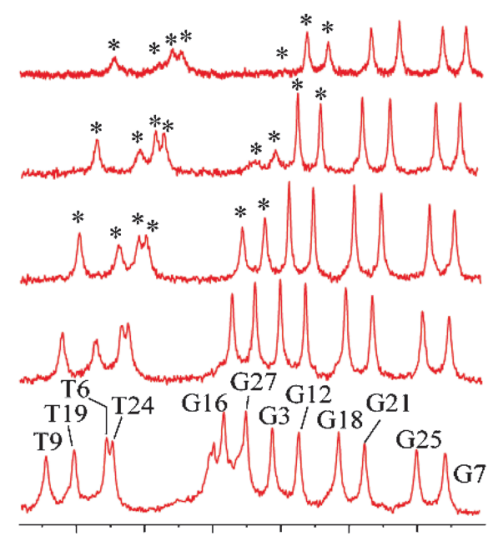

13.6

13.2

$\delta / \mathrm{ppm}$

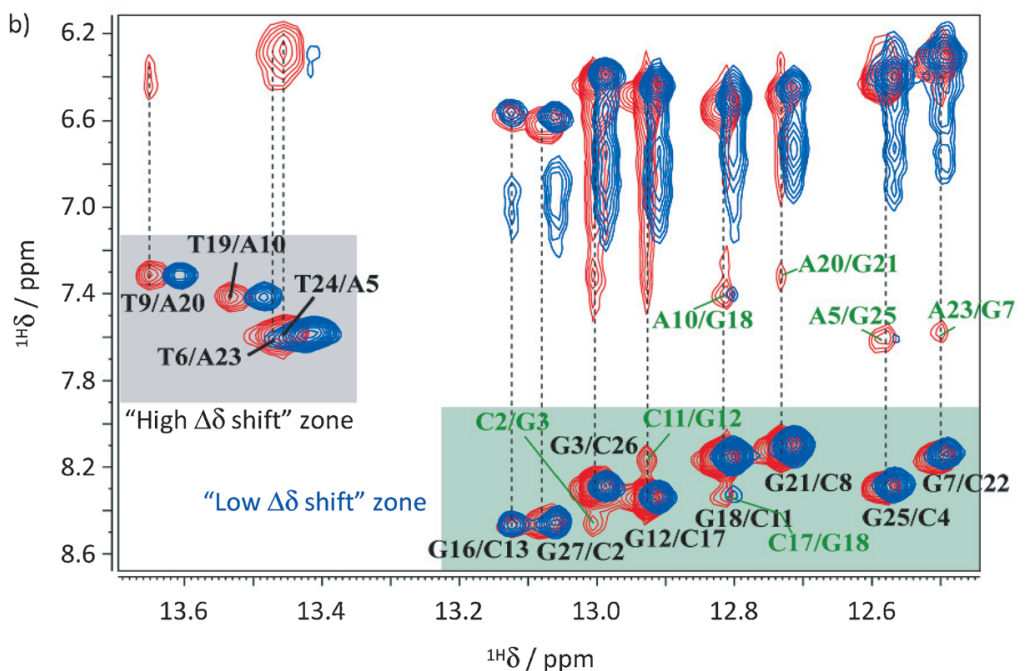

Figure 5. NMR spectroscopy study of the interaction between IR13 and DNA. a) 1D ${ }^{1} \mathrm{H}$ NMR spectra of the imino region of GG28 in the absence (black) and presence of IR13 (GG28/IR13 =1:1; red). b) 2D ${ }^{1} \mathrm{H}-{ }^{1} \mathrm{H}$ NOESY spectra of T-imino and G-imino base pairs of GG28 in the absence (red) and presence (blue) of IR13.

imino protons had decreased by a factor of 1.35 relative to that of free DNA, suggesting that complex formation attenuates high-frequency motions. In contrast, the $R_{2}$ value of the complex is increased by a factor of 1.4 relative to that of free DNA (Supporting Information, Figure S6), indicating that the peptide in its bound state has an increased overall correlation time. Furthermore, from the on-resonance $T_{1 \rho}$ measurements at spinlock field strengths of 3 and $6 \mathrm{kHz}$ for bound GG28, we could estimate a $R_{\mathrm{ex}}$ value of $8 \mathrm{~Hz} .^{[26-28]}$ The presence of residual $R_{\mathrm{ex}}$ indicates that GG28 and IR13 dynamically interact with each other on the fast-to-intermediate ( $\mu$ s-ms) NMR time scale.

To obtain further structural insight into the mechanism behind bacterial cell killing through the shutdown of genomic DNA by IR13, we first determined the $3 D$ structure of free GG28 by NMR spectroscopy and its docking structure bound to the IR13 peptide. Sequential assignment of the non-exchangeable base protons (purine $\mathrm{H} 8$ and pyrimidine $\mathrm{H} 6$ ) were performed by using through-space connectivities in combination with the $\mathrm{H}^{\prime}$-anomeric ribose protons in the $2 \mathrm{D}{ }^{1} \mathrm{H}-{ }^{1} \mathrm{H}$ NOESY

lation when trajectories were analyzed (Supporting Information, Figure S5). We also performed a series of $T_{1}, T_{2}$, and $T_{1 \rho}$ experiments for the imino protons of free GG28 and its complex with IR13, to understand the dynamics of GG28 at the atomic level. We found a remarkable decrease in the $R_{1}$ profile of T9 and T19 relative to that of the guanine bases, except for the terminal bases $\mathrm{G} 27$ and $\mathrm{G} 16$, which are highly dynamic due to their location at the termini (Supporting Information, Figure S6). Because all the A:T base pairs (T19, T9, T6, and T24) are located in the central region of GG28, we were unable to compare the $R_{1}$ profile of T9 and T19 with a reference "T" which is located away from the central region. The decreased dynamics of T9 and T19 in the complex form is a consequence of the strong hydrophobic interactions between the aromatic rings of Pro3-T9 or Trp8-T9 and Trp11-T19 (Supporting Information, Figure S6). Due to the signal overlap of imino protons of T6 and T24 of GG28, we did not consider the $R_{1}$ values of these residues to explain the actual dynamics (Supporting Information, Figure S6). Overall, the average $R_{1}$ value of the spectra (Figure 6 and Table S1). Strikingly, all the intra-residue and sequential NOEs observed between cytosine $\mathrm{H} 5$ and $\mathrm{H} 6$ and aromatic-aromatic protons $(\mathrm{H} 8-\mathrm{H} 5)$ were similar for both GG28 (Figure 6a) and its complex spectra (Figure $6 \mathrm{~b}$ ), providing evidence for normal base stacking in both duplexes. In addition, the intensities and chemical shifts of most of the resonances did not vary much between the control and complex DNA. This observation was further corroborated by the 2D ${ }^{1} \mathrm{H}-$ ${ }^{1} \mathrm{H}$ NOESY spectrum of the aromatic-aliphatic NOEs of GG28 in the free and complexed forms. In other words, sugar moieties do not influence structure stabilization. The NOE contacts for $\mathrm{H} 1^{\prime}-\mathrm{H}^{\prime} / \mathrm{H}^{\prime \prime}$ in both DNA structures (free and peptide-bound) were found to be isochronous (Supporting Information, Figure S7), suggesting that the sugar backbone conformation remains almost the same for both B-type DNAs.

The 3D structure of duplex DNA GG28 (PDB ID: 2M2C) was calculated using NMR-derived MD simulations with distance, dihedral, sugar, and base-pairing restraints (Figure 7). A total of 355 NOEs such as 90 strong, 113 medium, and 152 weak, 62 

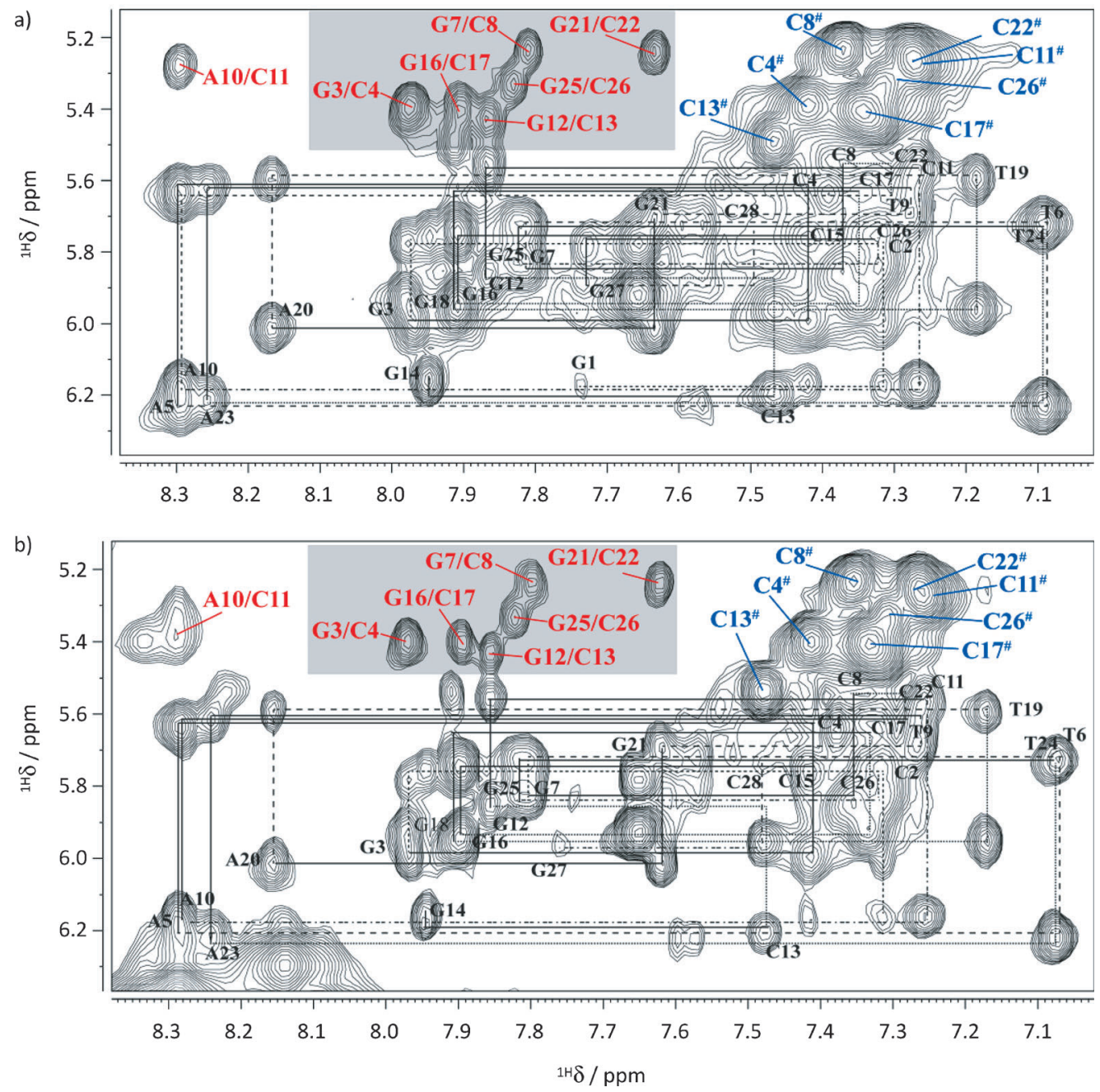

Figure 6. $2 \mathrm{D}{ }^{1} \mathrm{H}-{ }^{1} \mathrm{H}$ NOESY ( $500 \mathrm{MHz}, 298 \mathrm{~K}, 150 \mathrm{~ms}$ mixing time) spectra showing sequential assignments of the base $\mathrm{H} 6 / \mathrm{H} 8$ protons with $\mathrm{H} 1^{\prime}$-ribose protons of a) GG28 and b) GG28-IR13 complex (GG28/IR13=1:1).

Watson-Crick hydrogen bond, and a total of 192 torsional angles including $\alpha, \beta, \gamma, \delta, \varepsilon$, and $\xi$ were used for the structure calculation (Supporting Information Tables S2 and S3). The stereospecific assignment of sugar protons of a DNA duplex of this size is practically impossible due to severe signal overlap in getting the ${ }^{1} \mathrm{H}-{ }^{1} \mathrm{H}$ coupling constants. However, we obtained the correlation between $3^{\prime}-5^{\prime}$ phosphorus with $\mathrm{H} 5^{\prime} / \mathrm{H} 5^{\prime \prime}$ and $\mathrm{H}^{\prime}$, which led us to conclude that the $\gamma$ and $\xi$ dihedral angles exist in + and trans conformations, respectively (Figure S8). Simulated annealing in vacuum with the generalized Born model of DNA duplex with NMR-derived restraints were used to obtain an ensemble of eight stable conformations of B-type DNA structures with an RMSD of $0.7 \AA$ (Figure 7a). In general, the NMR-derived ensemble structure represents the average of the structure of a large number of molecules accumulated over an observed period of experimental time.

The 3D structure of IR13 (PDB ID: $1 \mathrm{G} 8 \mathrm{C}$ ) ${ }^{[17]}$ was then docked onto the solution structure of $\mathrm{GG} 28$ using the program Hex $6.3,{ }^{[29]}$ followed by MD simulation for a time period of 50 ns (Figure $7 \mathrm{~b}$ ). Representation of IR13 orientation over GG28 in the simulation time course is shown in Figure S9 (Sup- porting Information), where the B-form helicity of the duplex DNA is found to be well conserved in the complex system (Figure S10). The average RMSD of all the DNA residues from $50 \mathrm{~ns}$ MD suggests a stable GG28-IR13 complex (Figure S11). Alignment of free and IR13-bound structures yields $3.0 \AA$ RMSD because of the shift in the backbone dihedral in IR13-complexed DNA relative to that of the control. Amber-ff99SB force-fieldbased MD simulation predicted the RMSD for all the residues of the free state to be similar to or higher than that of the nucleobases in the complex state except for A5 in free duplex DNA. Structural perturbations of G14, C15, G16, C17, and G18 are much more dramatic in GG28, indicating that IR13 binds to the major groove of the DNA duplex (Figure S11 and Table S4). To correlate this observation, we performed UV spectroscopy of free GG28 and in complex with IR13 in the presence of methyl green, which preferentially binds to the major groove of duplex DNA. ${ }^{[30]}$ We also performed fluorescence assays with a minor-groove-binding fluorescent dye, 4',6-diamidino-2-phenylindole (DAPI). ${ }^{[31]}$ We observed a decrease in the UV absorbance peak for methyl green at $\sim 632 \mathrm{~nm}$ upon binding to duplex DNA. With successive additions of IR13 to the sample 
a)

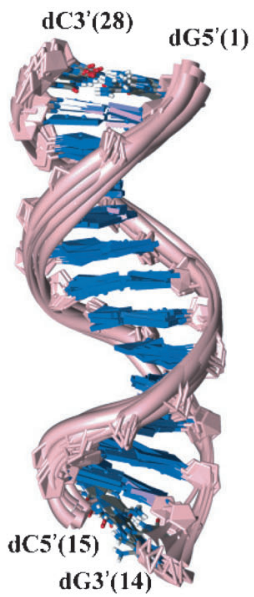

b)

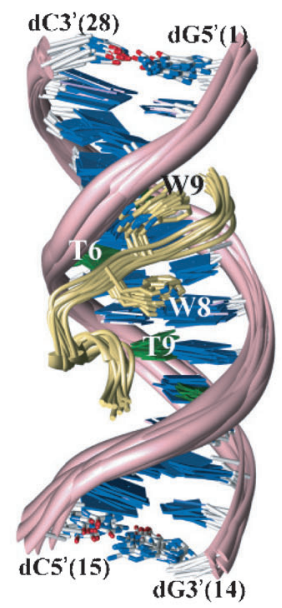

c)
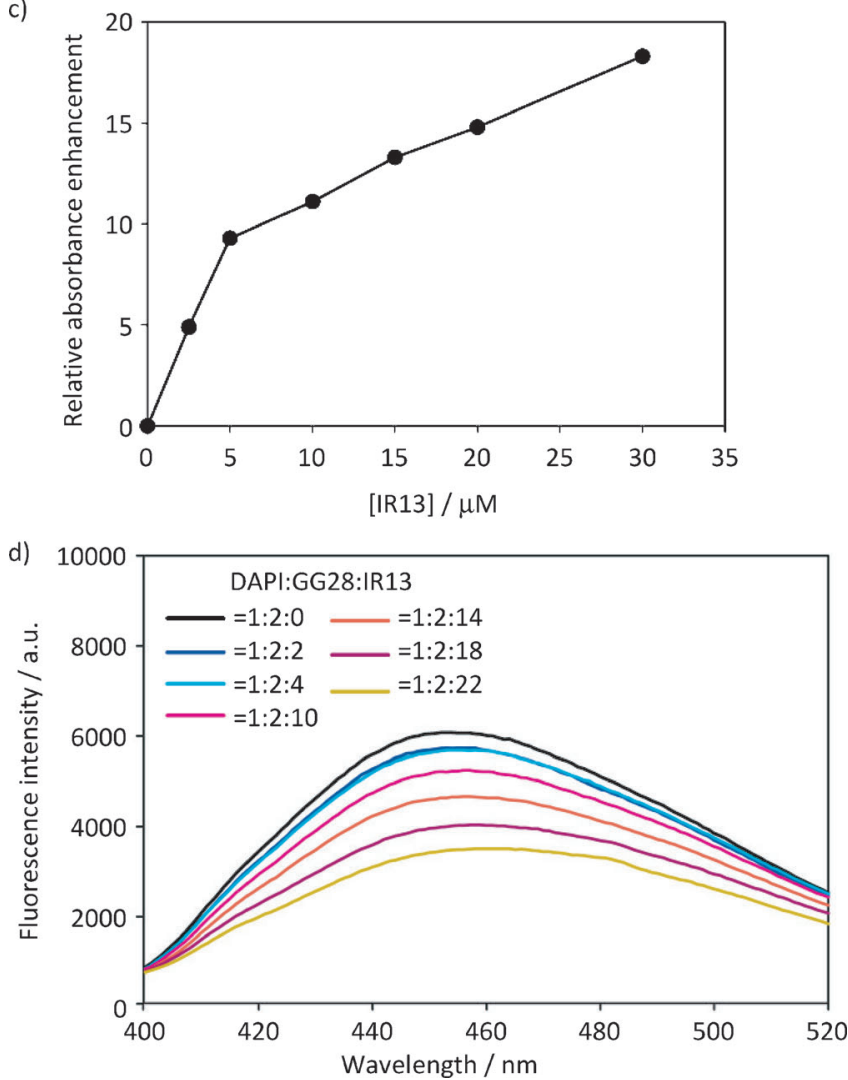

Figure 7. a) An ensemble of GG28 structures derived from NMR constraintsteered MD simulations. b) An ensemble of GG28-IR13 complex structures derived MD simulations. Residues Trp8 and Trp9 stabilize the DNA duplex structure by preventing solvation (images were produced with Chimera: http://www.rbvi.ucsf.edu/chimera/). c) Relative UV absorbance enhancement of methyl green with concomitant increase of IR13 from the GG28-methyl green complex, indicating IR13 binds to the major groove of duplex DNA due to its competition with methyl green. d) Fluorescence spectrum of DAPI mixed with GG28, at varied concentrations of IR13 to determine whether IR13 binds to the minor groove of duplex DNA.

containing GG28 and methyl green, the UV intensity of methyl green increased significantly. This demonstrates that IR13 displaces the methyl green dye from the major-groove-binding site of duplex DNA (Figure 7c). In contrast, a higher concentration of IR13 is required to compete with DAPI, which interacts

at the minor groove of duplex DNA (Figure $7 \mathrm{~d}$ ). Collectively, UV and fluorescence spectroscopy confirm that IR13 binds to the major groove of duplex DNA.

DNA is stabilized by binding to IR13, and residues Trp8 and Trp9 of IR13 play a particularly key role in stabilization of the duplex by desolvating the core of the DNA (Figure S12). This explanation is in good agreement with our thermal denaturation profile of GG28-IR13 and its analogues using CD spectroscopy (Figure $4 \mathrm{a}$ ). In binding, the peptide eventually wraps the DNA such that it cannot readily unwind (Figure $7 \mathrm{~b}$ ). The analysis of van't Hoff's isotherm (using CD melting data) of free DNA and its complexes with IR13 or analogues shows that complexation with IR13 increases the $\Delta \Delta G$ value of DNA from -9.1 to $-10.8 \mathrm{kcalmol}^{-1}$. Additionally, the magnitude of the enthalpy and entropy of the GG28-IR13 complex increases from -22.0 to $-31.4 \mathrm{kcal} \mathrm{mol}^{-1}$ (for enthalpic change) and from -43.4 to $-69.2 \mathrm{cal} \mathrm{mol}^{-1} \mathrm{~K}^{-1}$ (for entropy change), respectively, favoring the complex formation thermodynamically (Supporting Information, Table S5). A similar scenario was observed for the GC28 duplex bound to IR13. Taken together, the of the thermodynamic stability of these complexes was found to increase following the rank order: GG28-IR13 $\gg$ GG28IR13HH $\geq$ GG28-IR13FF > GG28-IR13AA > GG28. In fact, both CD and NMR studies revealed that the DNA is stabilized by interaction with IR13.

In conclusion, we have shown that IR13 binds and wraps duplex DNA so that it cannot unwind easily. This leads to the subsequent inhibition of DNA replication and transcription. Through various spectroscopic and microscopy techniques we also found that the PWWP motif is crucial for binding of IR13 to GG28. We are currently in the process of using isotopically $\left({ }^{15} \mathrm{~N}\right.$ and $\left.{ }^{13} \mathrm{C}\right)$ labeled peptides to refine structure in complex with duplex DNA. We hope that the high-resolution structure of the indolicidin-DNA complex presented herein will open up new possibilities to modify IR13 to enhance its antibacterial potency while diminishing its cytotoxic effects.

\section{Experimental Section}

Detailed experimental methods are provided in the Supporting Information.

\section{Acknowledgements}

A.B. thanks DST Fast Track (SERC/LS-527/2011, Government of India) for financial support. S.C. and S.G. thank DST, Government of India for a Ramanujan Fellowship. S.G. thanks CSIR-IICB Kolkata for financial support. A.G., J.J., A.S., B.J., and R.K.K. thank CSIR, Government of India for fellowships. A.B. thanks Prof. Dr. A. Ramamoorthy (University of Michigan, USA) for the relaxation pulse program and is grateful to Prof. Dr. Dr. Hans-Christian Siebert (RIB-NT, Kiel, Germany), Prof. Dr. A. Ramamoorthy (University of Michigan), and Prof. Dr. Adolf Gogoll (Uppsala University, Sweden) for critical reading and suggestions for this manuscript. The Central Instrument Facility (CIF) of Bose Institute is gratefully acknowledged for peptide synthesizer, $C D$, fluorescence, and NMR 
instrument facilities. Atomic coordinates of the structures of GG28 have been deposited at the RCSB Protein Data Bank (accession number $2 \mathrm{M} 2 \mathrm{C}$ ).

Keywords: AMP • duplex DNA • indolicidin • molecular dynamics - NMR spectroscopy

[1] K. M. O'Connell, J. T. Hodgkinson, H. F. Sore, M. Welch, G. P. Salmond D. R. Spring, Angew. Chem. Int. Ed. 2013, 52, 10706-10733; Angew. Chem. 2013, 125, 10904-10932.

[2] M. Zasloff, Nature 2002, 415, 389-395.

[3] G. Taubes, Science 2008, 321, 356.

[4] R. I. Lehrer, Nat. Rev. Microbiol. 2004, 2, 727-738.

[5] M. Torrent, J. Valle, M. Nogués, E. Boix, D. Andreu, Angew. Chem. Int. Ed. 2011, 50, 10686-10689; Angew. Chem. 2011, 123, 10874-10877.

[6] E. Giralt, Small Wonders: Peptides for Disease Control (Eds.: K. Rajasekaran, J. W. Cary, J. M. Jaynes, E. Montesinos), Wiley Online Library, 2013.

[7] A. Bhunia, R. Saravanan, H. Mohanram, M. L. Mangoni, S. Bhattacharjya, J. Biol. Chem. 2011, 286, 24394-24406.

[8] A. Bhunia, A. Ramamoorthy, S. Bhattacharjya, Chem. Eur. J. 2009, 15, $2036-2040$.

[9] A. Bhunia, H. Mohanram, P. N. Domadia, J. Torres, S. Bhattacharjya, J. Biol. Chem. 2009, 284, $21991-22004$.

[10] D. Zweytick, G. Deutsch, J. Andrä, S. E. Blondelle, E. Vollmer, R. Jerala, K. Lohner, J. Biol. Chem. 2011, 286, 21266-21276.

[11] A. W. Young, Z. Liu, C. Zhou, F. Totsingan, N. Jiwrajka, Z. Shi, N. R. Kallenbach, MedChemComm 2011, 2, 308-314.

[12] M. L. Mangoni, R. F. Epand, Y. Rosenfeld, A. Peleg, D. Barra, R. M. Epand, Y. Shai, J. Biol. Chem. 2008, 283, 22907-22917.

[13] M. Zanetti, J. Leukocyte Biol. 2003, 75, 39-48.

[14] M. Son, Y. Lee, H. Hwang, S. Hyun, J. Yu, ChemMedChem 2013, 8, 1638 1642

[15] R. K. Sharma, S. Sundriyal, N. Wangoo, W. Tegge, R. Jain, ChemMedChem 2010, 5, 86-95.
[16] M. E. Selsted, M. J. Novotny, W. L. Morris, Y.-Q. Tang, W. Smith, J. S Cullor, J. Biol. Chem. 1992, 267, 4292-4295.

[17] A. Rozek, C. L. Friedrich, R. E. Hancock, Biochemistry 2000, 39, 15765 15774.

[18] J. E. Shaw, J.-R. Alattia, J. E. Verity, G. G. Privé, C. M. Yip, J. Struct. Biol. 2006, 154, 42-58

[19] C. Subbalakshmi, N. Sitaram, FEMS Microbiol. Lett. 1998, 160, 91 - 96.

[20] C. Qiu, K. Sawada, X. Zhang, X. Cheng, Nat. Struct. Mol. Biol. 2002, 9, 217-224.

[21] A. Vezzoli, N. Bonadies, M. D. Allen, S. M. Freund, C. M. Santiveri, B. T. Kvinlaug, B. J. Huntly, B. Göttgens, M. Bycroft, Nat. Struct. Mol. Biol. 2010, 17, 617-619.

[22] C. Subbalakshmi, V. Krishnakumari, R. Nagaraj, N. Sitaram, FEBS Lett. 1996, 395, 48-52.

[23] C.-H. Hsu, C. Chen, M.-L. Jou, A. Y.-L. Lee, Y.-C. Lin, Y.-P. Yu, W.-T. Huang, S.-H. Wu, Nucleic Acids Res. 2005, 33, $4053-4064$.

[24] A. Biswas, A. Saha, B. Jana, P. Kurkute, G. Mondal, S. Ghosh, ChemBioChem 2013, 14, 689-694.

[25] M. Bhagawati, S. Ghosh, A. Reichel, K. Froehner, T. Surrey, J. Piehler, Angew. Chem. Int. Ed. 2009, 48, 9188-9191; Angew. Chem. 2009, 121, $9352-9355$

[26] D. Davis, M. Perlman, R. London, J. Magn. Reson. Ser. B 1994, 104, 266 275.

[27] C. Deverell, R. Morgan, J. Strange, Mol. Phys. 1970, 18, 553-559.

[28] A. Palmer III, C. D. Kroenke, J. P. Loria, Methods Enzymol. 2001, 339, 204 238.

[29] D. W. Ritchie, Proteins Struct. Funct. Bioinf. 2003, 52, 98-106.

[30] E. Tuite, U. Sehlstedt, P. Hagmar, B. Nordén, M. Takahashi, Eur. J. Biochem. 1997, 243, 482-492.

[31] N. Spacková, T. Cheatham III, F. Ryjácek, F. Lankas, L. Van Meervelt, P. Hobza, J. Sponer, J. Am. Chem. Soc. 2003, 125, 1759-1769.

Received: June 14, 2014

Published online on July 17, 2014 